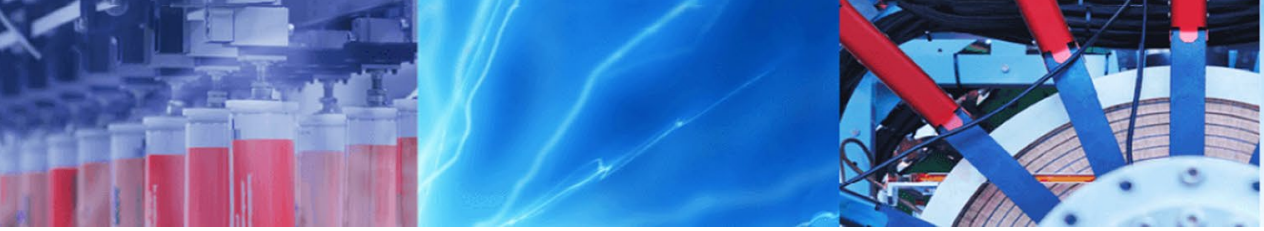

Research Article

\title{
Burst mode versus single-pulse machining for Bessel beam micro-drilling of thin glass: study and comparison
}

\author{
V. V. Belloni ${ }^{1} \cdot$ V. Sabonis ${ }^{2} \cdot$ P. Di Trapani ${ }^{1} \cdot$ O. Jedrkiewicz ${ }^{3}$ (i)
}

Received: 13 May 2020 / Accepted: 12 August 2020 / Published online: 29 August 2020

(c) Springer Nature Switzerland AG 2020

\begin{abstract}
We present a study on the micro-drilling process by means of a picosecond Bessel-Gauss beam, and the achievements obtained on a 200- $\mu$ m-thick AF32 glass sample in different laser fabrication regimes. In particular, we compare the results and morphology of the holes generated with a high-repetition-rate pulsed laser, respectively, in the single-pulse mode and in the burst mode machining regimes. We highlight the advantages or drawbacks of these two types of microfabrication for the generation of through-holes. For a given pulse density, the burst mode turns out to be advantageous with respect to the single-pulse mode fabrication in terms of lower energy per pulse needed and higher speed of drilling, even if the stronger thermal effects can more easily lead to surface cracks. On the other hand, by adjusting the pulse density below a critical level, it can be shown that the single-pulse regime can be adopted for the generation of more regular through-holes and cleaner apertures, even if multiple pass operation is likely to be needed.
\end{abstract}

Keywords Laser fabrication · Micro-hole drilling $\cdot$ Single-pulse machining $\cdot$ Burst mode machining $\cdot$ Bessel beams

\section{Introduction}

Processing of materials by ultrashort laser pulses has undergone a fast evolution over the last decade, and ultrafast laser systems have been widely applied in many scientific and industrial fields [1, 2]. In particular, the laser microfabrication of dielectrics takes advantage of the interaction of ultrashort high-intensity pulses with the material to generate 2D and 3D structures in the processed sample. It relies on the strong nonlinear absorption process of energy taking place only in the highly localized focus of the beam, and enabling a structural modification such as a refractive index change or the creation of voids and ablations inside the material, with very high precision [3]. The advantage of using femtosecond or picosecond laser sources lies indeed in the ability to deposit energy into the material before thermal diffusion occurs, resulting in the reduction of heat-affected zones [4].

Due to the industrial demand for high-throughput laser process, major efforts have been recently addressed to the development of ultrafast laser sources with increasing average powers and repetition rates. Nowadays, industrial ultrashort pulsed lasers are commercially available with average powers in the range of $100 \mathrm{~W}$, pulse energies up to $400 \mu \mathrm{J}$ and repetition rates up to several $\mathrm{MHz}$ [5]. The increase in average power and repetition rate of the ultrafast laser has led to improved throughput and industrial productivity together with the occurrence of some problems. In fact, the expected advantages in terms of higher quality and efficiency can be counterbalanced by other drawbacks such as heat accumulation. The latter occurs because pulse after pulse, fractions of laser energy released onto the target in the form of heat lead

O. Jedrkiewicz, ottavia.jedrkiewicz@ifn.cnr.it | 'Dipartimento di Scienza e Alta Tecnologia, Università degli Studi dell'Insubria, Via Valleggio 11, 22100 Como, Italy. ${ }^{2}$ UAB Altechna Rnd, Vilnius, Lithuania. ${ }^{3} \mathrm{CNR}$, Istituto di Fotonica e Nanotecnologie, Udr di Como, Via Valleggio 11, 22100 Como, Italy. 
to irreversible thermal damage, such as cracks or melting, which worsen the quality and precision of the manufacturing process. One solution to overcome the issues related to heat accumulation when using high-repetition-rate lasers has been to employ the so-called burst mode (BM) processing. The latter consists in using sequences (or bursts) of several ultrashort laser pulses with inter-pulse time delay shorter than $1 \mu \mathrm{s}$ [6]. The most interesting studies conducted on BM processing with inter-pulse time delay on the nanosecond scale showed that under some experimental conditions, bursts improved the heat-related issues $[7,8]$.

In the present paper, we shall make a comparative study between the standard single-pulse laser processing and the BM laser processing applied to a particular case of micromachining of transparent dielectric samples, i.e., micro-hole drilling by means of finite-energy pulsed Bessel beams $[9,10]$ The ultrashort laser microfabrication technique has recently found great interest for material processing such as micro-hole drilling and cutting, and such machining processes have indeed been recently applied in thin glass samples by using "non-diffracting" Bessel-Gauss beams [11-15]. The latter have cylindrical symmetry and are featured in the transverse cross section by an intense central spot surrounded by weaker concentric rings, with the advantage that their use allows an easier manipulation of the diameter and depth of the microchannels or microholes to be created [16-21]. Moreover, a sample translation along the beam propagation direction for in-depth microstructuring is not necessarily needed thanks to the elongated focal length of the $B B$, in contrast to the use of standard processing techniques that employ focused Gaussian pulses combined with a sample translation in three dimensions often requiring multiple shot machining. It is worth noting that finite-energy Bessel beams (BB) have been the object of intense research and applications in different fields, thanks to their self-reconstruction properties and their capacity of maintaining a smooth longitudinal fluence profile under both linear propagation and nonlinear propagation [22].

Our work focuses on the study of the laser micro-drilling process and the observation of the through-holes obtained in a $200-\mu m$-thick glass sample, in two different laser fabrication regimes, by means of a picosecond Bessel-Gauss beam. The machining process is based on the combination of such non-diffracting beams with a trepanning-like technique, already used for the etching of glass-air interfaces and for preliminary results on microhole drilling in thin glass samples [15]. Different parameters and their effect such as the number of writing circular trajectories, the fabrication speed, the pulse energy and the pulse density are studied and optimized. In particular, we compare the results and the morphology of the holes obtained with a high-repetition-rate laser in the singlepulse mode and in the burst mode machining regime, respectively, highlighting advantages and drawbacks of these two microfabrication regimes in the context of the BB hole drilling process.

\section{The experiment}

The laser microfabrication technique used to drill round through-holes in thin glasses, without the need of chemical etching, is based on the use of a zero-order pulsed BB impinging orthogonally to the sample surface combined with spiraling writing trajectories as in a trepanning process [15]. The experimental setup is divided into two parts, consisting in the micromachining part and the sample imaging part. A scheme of the setup is shown in Fig. 1. The $B B$ is generated by means of a fused silica axicon, with base angle $\gamma=1^{\circ}$ and refractive index $n_{\mathrm{ax}}=1.45$. Given the laser input beam size, the BB is typically featured by a $50 \mu \mathrm{m}$ core size and $60 \mathrm{~cm}$ Bessel zone length (non-diffracting length); thus, in order to drill a few hundred $\mu \mathrm{m}$ thick sample, it is demagnified by a telescope that is composed of a lens (with focal length $f_{1}=300 \mathrm{~mm}$ ) and a microscope objective (focal length, $f_{\text {obj }}=9 \mathrm{~mm}$ ). The resulting beam having a cone angle of $15^{\circ}$ is featured by a core of $1.5 \mu \mathrm{m}$ and a Bessel zone of the order of $460 \mu \mathrm{m}$. The beam is then deflected and sent vertically down toward the dielectric sample positioned on a 3D motorized stage which can be moved in all directions with micrometer precision and is driven by the software SCA, System Control Application (Altechna Rnd). A dichroic mirror is positioned between the lens and the objective, in such a way to allow an online imaging on a CCD camera of the sample, back-illuminated by a red light. The laser pulse energy is modified by means of neutral density filters. Note that the final beam features are characterized in air before the micromachining process by means of an imaging system (not shown).

The experiments have been performed by using a highrepetition-rate infrared pulsed laser (Picoblade2, Lumentum). This laser delivers pulses at $1064 \mathrm{~nm}$ with a fixed pulse width of $10 \mathrm{ps}$ and a laser repetition rate (LRR) of $200 \mathrm{kHz}$. It can operate in single-pulse mode or in burst mode. The single-pulse mode generates a single pulse every $\Delta \tau=\mathrm{LRR}^{-1} \mathrm{~s}$, with a maximum energy arriving at the sample of $150 \mu \mathrm{J}$ (taking into account the beam path losses), and the burst mode produces packets of closely distributed pulses separated by $\Delta t$. In the same packet of pulses, the distance between pulses is given by $\Delta \tau_{B}$ (see Fig. 2) which can be equal to $12 \mathrm{~ns}$ or $156 \mathrm{~ns}$, respectively, depending on the burst mode used in our experiments. The maximum burst package energy arriving at the sample in this operating regime ranges from 290 to $320 \mu \mathrm{J}$ 
Fig. 1 Scheme of the experimental setup typically used for Bessel beam micromachining
Fig. 2 Scheme of the pulsed laser operating modes

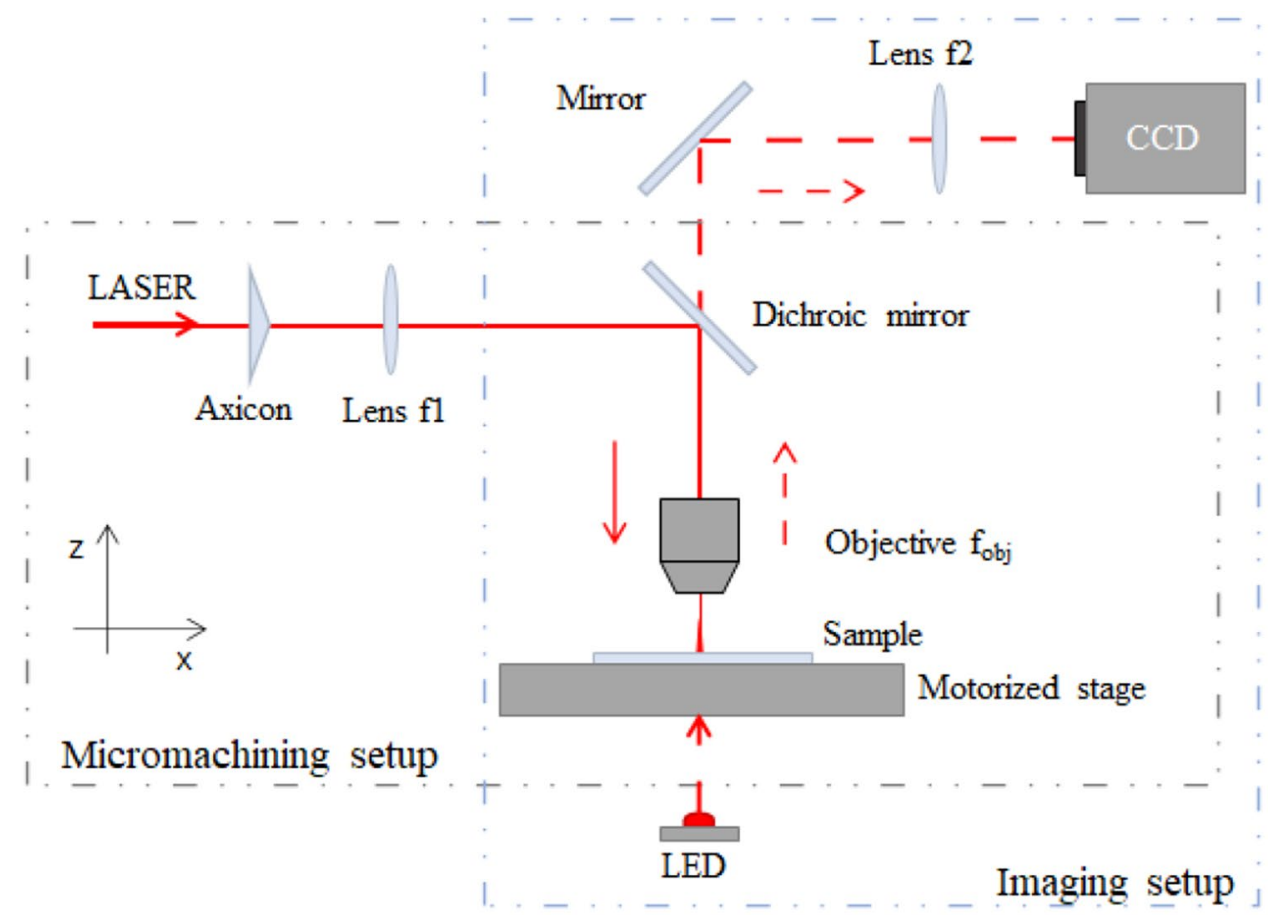

a)

Single pulse mode

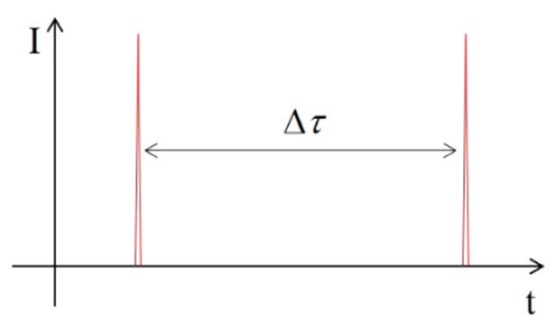

b)

Burst mode

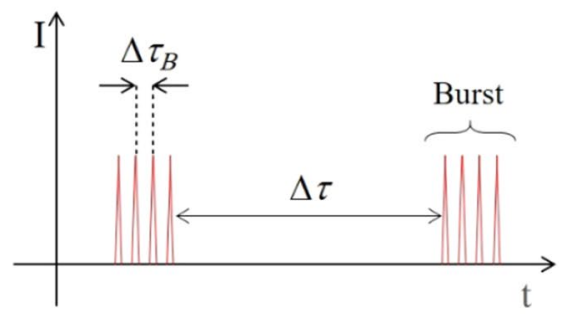

depending on the burst type used, as we shall see in the following. Note that the energy values reported throughout this paper have been measured with a maximum uncertainty due to laser fluctuations of about $1 \%$.

The software SCA allows the design of writing patterns for the micromachining of the considered materials. Note that in our setup, as described above, it is the sample which is moved with respect to the Bessel beam. For our drilling experiments, the writing trajectory is spiraling as described in Fig. 3. It is interesting to note that different parameters can affect the hole quality and morphology: the distance between circles (denoted in the following by "step"), the number of circles, the density of pulses (pulses $\mathrm{mm}^{-1}$ ), the speed of fabrication and the relative position between the Bessel beam and the sample thickness. The distance between the writing circles (or more precisely semicircles as shown in Fig. 3 ) affects the radial density of the impinging pulses. Changing the number of circles modifies the size of the holes, with a minimum

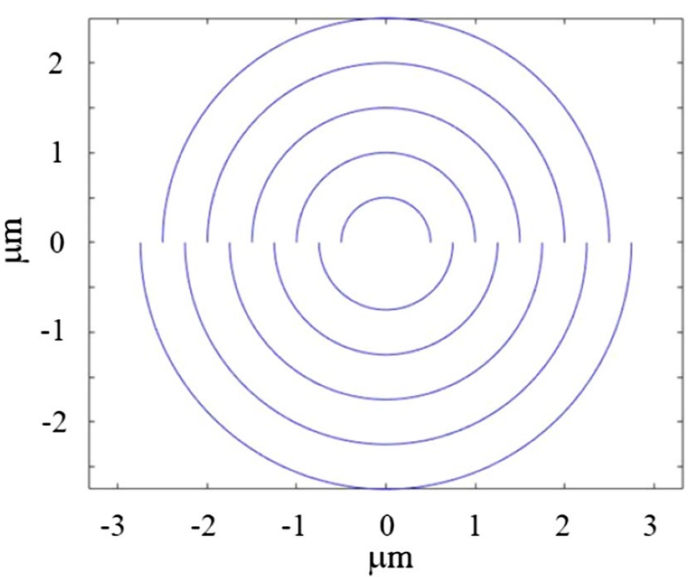

Fig. 3 Example of spiraling writing trajectories programmed with SCA for the hole drilling 
needed number (for a maximum aspect ratio of the hole); this number also depends on the thickness of the sample to drill. It is worth noting that the density of pulses, the speed of fabrication and the laser repetition rate are linked together. While for low repetition rate high power laser systems with fixed repetition rate, the speed determines the pulse density according to

Pulse density $=\mathrm{LRR} /$ speed $=1 /($ speed $\cdot \Delta t)$

in the high-repetition-rate regime with a beam delivery featured by a chosen fixed pulse density as for the Picoblade laser case, we can calculate an equivalent repetition rate (ERR) as

$\operatorname{ERR}=\Delta t^{-1}=$ speed $\cdot$ pulse density

and therefore the temporal distance between two consecutive pulses can be modified by changing the speed or/ and the pulse density. Note that in most of our machining tests, as shown in the following, the pulse density (which tells us the number of laser shots that must be contained within a given spatial distance) is kept fixed and the same, in both the single-pulse and the BM drilling modes. In both operating laser regimes, this is ensured by software and hardware interfaces between the laser and the motorized stage guaranteeing a constant synchronization between the arrival of the single pulses (or bursts) and the stage movement.

Finally, the relative position between the Bessel zone and the sample has to be set in order to optimize the beam interaction along the whole sample thickness. This is done by positioning the $\mathrm{BB}$ in such a way that the Bessel zone (Bessel intensity profile along propagation) is symmetrically distributed along the sample thickness, i.e., with the maximal intensity in the middle of the bulk.

\section{Single-pulse micromachining mode}

In this work, we have analyzed the possibility to drill, by means of picosecond Bessel beams and in two different laser fabrication regimes through-holes in a $200-\mu \mathrm{m}$-thick AF 32 glass sample featured by a low thermal expansion coefficient. The study presented here extends previous preliminary work on the etching and micro-hole drilling with Bessel beams [15], and by presenting new results, it allows to make a comparison between the achievements obtained in the single-pulse machining regime and those obtained in the BM regime. In both cases, several tests have been performed by modifying different fabrication parameters. In this section, we present results obtained in the standard single-pulse microfabrication configuration.

\subsection{BB spiraling hole drilling with fixed pulse density}

First experimental tests were performed in the singlepulse machining mode regime in order to generate microholes in similar conditions to those of the previous work [15]. The pulse energy used was about $100 \mu \mathrm{J}$, and taking into account the dimension of the Bessel core and the laser pulse duration (10 ps), we can estimate a pulse peak intensity of about $10^{14} \mathrm{~W} \cdot \mathrm{cm}^{-1}$, enough to trigger the photoionization process. The typical machining parameters include a fabrication speed of $0.8 \mathrm{~mm} \mathrm{~s}^{-1}, 60$ writing circles, with a spatial step between them of $0.25 \mu \mathrm{m}$, and a chosen pulse density of 5000 pulses $\mathrm{mm}^{-1}$. Note that initially the glass sample was drilled several times, by running at the same position the whole spiraling writing trajectory more than once, in order to identify with the available energy the minimum number of processing passes needed for a through-hole generation. In Fig. 4, we show the results obtained in one machining pass and two machining passes, respectively: one pass led to the formation of a through-hole with very different apertures sizes at the top and bottom surfaces, attributed to an insufficient pulse energy, while the test with two writing passes has shown the possibility to generate even with energy limitation a well-defined and more homogeneous through-hole. Tests with a greater number of writing passes did not lead to any substantial difference with respect to the latter case, so in the following experiments the holes are generated with two machining passes. The glass was drilled along its thickness near the edge of the sample, allowing us after the fabrication to analyze with a microscope objective the morphology of the hole along the whole sample thickness. We can observe that the entrance aperture is bigger than the exit one and it has a slight taper shape. Note for
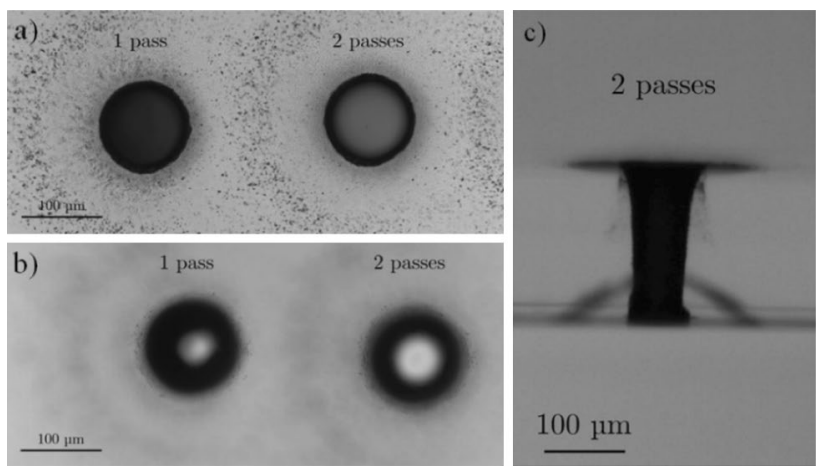

Fig. 4 Optical microscope images of micro-holes performed in a 200- $\mu$ m-thick AF32 glass in one or two writing passes, in the standard single-pulse machining regime. a Top surface of the sample, b bottom surface, $\mathbf{c}$ lateral view of the through-hole drilled in two writing passes. Speed of fabrication $0.8 \mathrm{~mm} \mathrm{~s}^{-1}$, pulse density 5000 pulses $\mathrm{mm}^{-1}, 60$ writing circles 
completeness that with the same trepanning technique and fabrication parameters, it is possible to generate larger holes as for instance the millimeter size diameter hole shown in Fig. 5. The spiraling trajectory (with 60 circles) was performed only within a $50-\mu \mathrm{m}$-thick corona with radius equal to the radius of the desired aperture. In other words, in this case, instead of starting the spiraling laser machining from the center of the scheme shown in Fig. 3, the beam can be initially positioned on the semicircular trajectory which lies at a distance approximately equal to the radius of the $\mathrm{mm}$-sized hole to be drilled. The 60 writing circles can then be processed from that point with the result that only a $50-\mu \mathrm{m}$-thick corona will be effectively machined by the BB. It is important to point out that there is therefore no need to machine the whole internal aperture area when holes of dimensions much larger than the sample thickness have to be drilled. In this case, after the two-pass laser writing process the internal part of the machined sample falls down spontaneously.

\subsection{Dependence on the number of circular writing trajectories}

With the same fabrication parameters, we investigated the dependence of the micro-hole size on the number of writing circles used during the trepanning process. The test was performed with a number of circles going from 40 to 60 by steps of 5 and the results are shown in Fig. 6 . The apertures diameters (in the range of 60-90 $\mu \mathrm{m}$ ) both at the top and bottom surfaces increase with the number of circles, but with different rates relatively to the two surfaces. Moreover, the exit apertures are always smaller than the entrance apertures. We note nevertheless that the holes tend to be more regular when increasing the

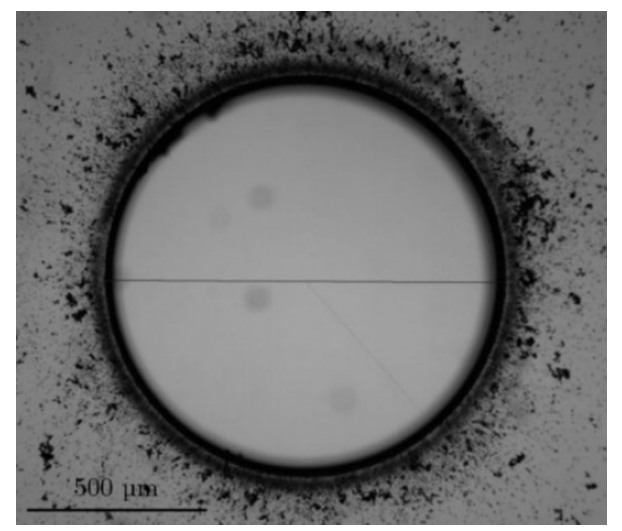

Fig. 5 Optical microscope image of a millimeter diameter hole generated in a $200-\mu m$-thick AF32 glass with two writing passes, in the standard single-pulse machining regime. Top view of the sample. Speed of fabrication $0.8 \mathrm{~mm} \mathrm{~s}^{-1}$, and pulse density 5000 pulses $\mathrm{mm}^{-1}, 60$ writing circles in the machined area

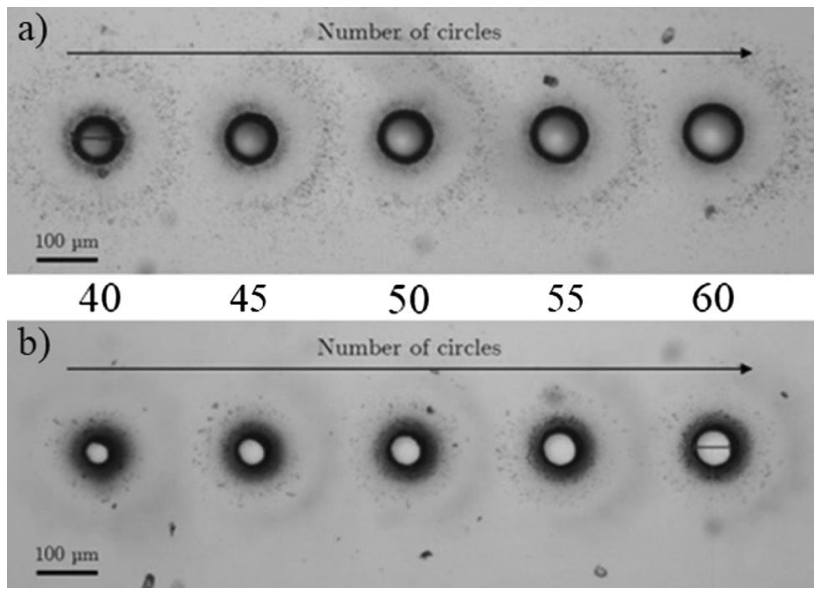

Fig. 6 Dependence of the hole diameters on the number of writing circles during the fabrication in single-pulse machining regime (from 40 to 60 circles by step of 5). Optical microscope images of the top (a) and bottom (b) surfaces of the 200- $\mu$ m-thick AF32 glass sample. Speed of fabrication $0.8 \mathrm{~mm} \mathrm{~s}^{-1}$ and pulse density 5000 pulses $\mathrm{mm}^{-1}$

number of circles, as we can see in the plot of the difference of the diameters (Fig. 7). The results have also shown the possibility of generating through-holes and thus with a minimum number of circular writing trajectories (in our particular case, this was 40 ) and thus a minimum diameter that depends on the sample thickness.

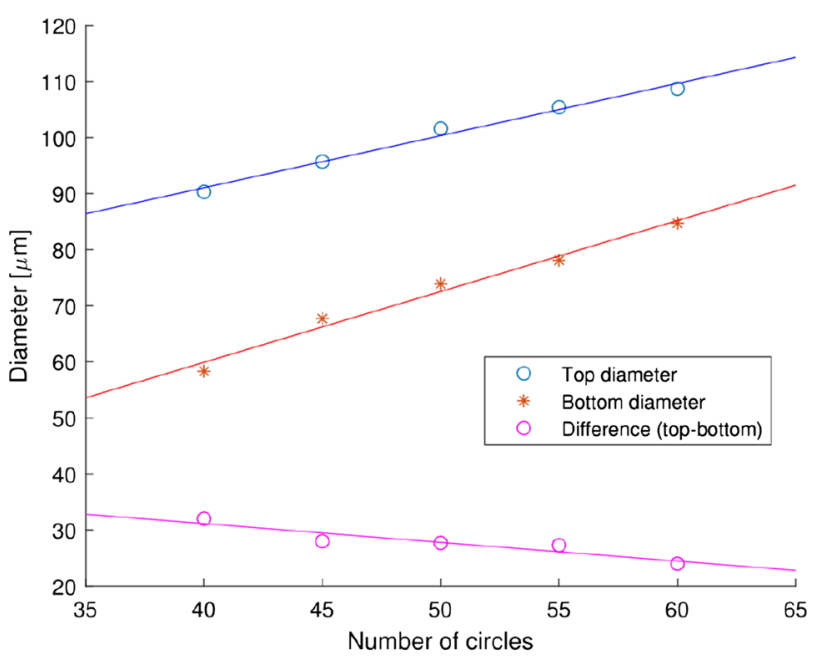

Fig. 7 Plots of the diameters of the generated holes as a function of the number of writing circles relatively to the top and the bottom surface, respectively, and difference between the two diameters. The uncertainty in the measurements is given by the size of the data points 


\subsection{Effect of the fabrication speed in single-pulse machining regime}

The next step has been to study the dependence of the micro-holes morphology and quality on the speed of fabrication. As we have seen in the previous section, for a given laser pulse density set in the machining process, the speed of fabrication (i.e., the motorized sample holder speed) influences the temporal distance between the pulses. Indeed setting the laser source in density mode implies that the repetition rate of the laser is automatically set in such a way to have the same number of pulses impinging locally on the sample. In other words, the larger the fabrication speed, the higher the laser repetition rate. In Table 1, we have reported the temporal distances between pulses and the estimated fluences for different speeds of fabrication in our experiment. Note that the fluence was calculated as

Fluence $=(E \times N) / A$

by evaluating the number $N$ of arriving pulses and the total area $A$ covered by the pulses (i.e., by the partially

Table 1 Temporal delay $\Delta \tau$ between arriving pulses and estimated fluences evaluated over an arbitrary time interval of $250 \mu \mathrm{m}$, as a function of the fabrication speed, in the regime of fixed density mode processing (5000 pulses $\mathrm{mm}^{-1}$ )

\begin{tabular}{lll}
\hline $\begin{array}{l}\text { Speed } \\
{\left[\mathrm{mm} \mathrm{s}^{-1}\right]}\end{array}$ & $\begin{array}{l}\Delta \tau \\
{[\mu \mathrm{s}]}\end{array}$ & $\begin{array}{l}\text { Fluence } \\
{\left[10^{3} \mathrm{~J} \mathrm{~cm}^{-2}\right]}\end{array}$ \\
\hline 0.8 & 250 & 1.4 \\
1.8 & 111 & 2.9 \\
2.8 & 71 & 4.1 \\
3.8 & 53 & 5.1 \\
4.8 & 42 & 6.0 \\
5.8 & 34 & 6.7 \\
\hline
\end{tabular}

superimposed BB cores) within the same time interval of $250 \mu \mathrm{s}$, where $E$ is the pulse energy. The chosen time interval for the estimation of the radiation fluence is the maximum temporal window in order to have only one laser shot in the case of lowest fabrication speed $\left(0.8 \mathrm{~mm} \mathrm{~s}^{-1}\right)$. Clearly, the number of total arriving pulses increases linearly with the speed of fabrication. The area of incidence was calculated by taking into consideration the superposition of the spatial cores of the pulses. Thus, the radiation fluence on the sample increases with the speed, while the temporal distance between two consecutive pulses decreases. The effect of the fabrication speed on the drilling process can be observed in Fig. 8 . As we can see in the optical microscope images, the apertures present a good quality both at the top and bottom surfaces for speeds up to $2.8 \mathrm{~mm} \mathrm{~s}^{-1}$. For higher fabrication speeds, cracks and rough edges are evident in all the samples. This change in behavior may be explained by thermal effects occurring during the machining process. A decrease in the temporal distance between pulses can lead to higher thermal accumulation effects, implying that the pulses at their arrival on the sample interact with a soft and hot matter instead of a cold and re-solidified one. The increase in temperature of the modified zone causes a higher pressure against the surrounding area that could lead to unpredictable cracks. These results suggest that a complete cooling of the heataffected region of our glass sample occurs in few tens of $\mu \mathrm{s}$.

In order to study the internal morphology of a hole drilled with speed $2.8 \mathrm{~mm} \mathrm{~s}^{-1}$, we performed a microfabrication across the lateral edge of the glass sample (semi-hole). Figure 9 shows the top (a), the bottom (b) and a lateral view $(c, d)$ of the hole observed under the
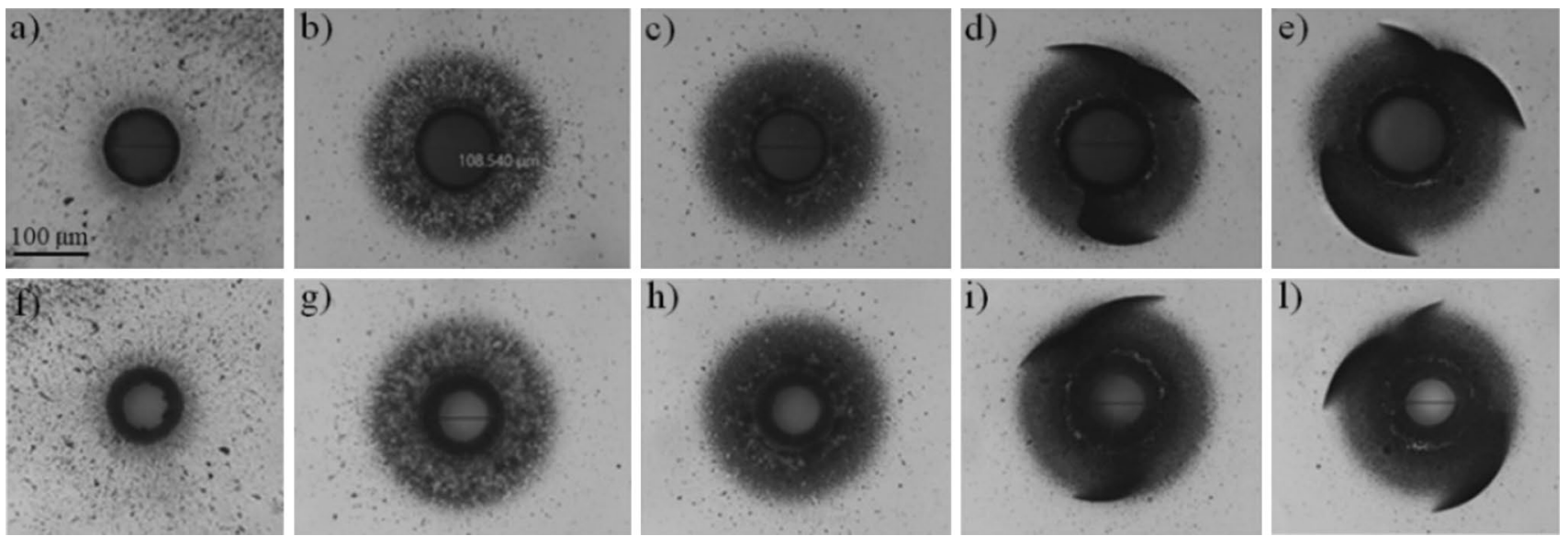

Fig. 8 Optical microscope images of holes drilled with 60 writing circles in density mode (5000 pulses $\mathrm{mm}^{-1}$ ) presented as a function of the speed of fabrication. The top row refers to the images of the top surface of the sample, while the bottom row refers to the images of the bottom surface. The fabrication speeds are $0.8 \mathrm{~mm} \mathrm{~s}^{-1}$ (a) and (f); $1.8 \mathrm{~mm} \mathrm{~s}^{-1}(\mathbf{b})$ and $(\mathbf{g}) ; 2.8 \mathrm{~mm} \mathrm{~s}^{-1}(\mathbf{c})$ and (h); $3.8 \mathrm{~mm} \mathrm{~s}^{-1}$ (d) and (i); and $4.8 \mathrm{~mm} \mathrm{~s}^{-1}$ (e) and (I). All images have the same scale bar $(100 \mu \mathrm{m})$ as in (a) 

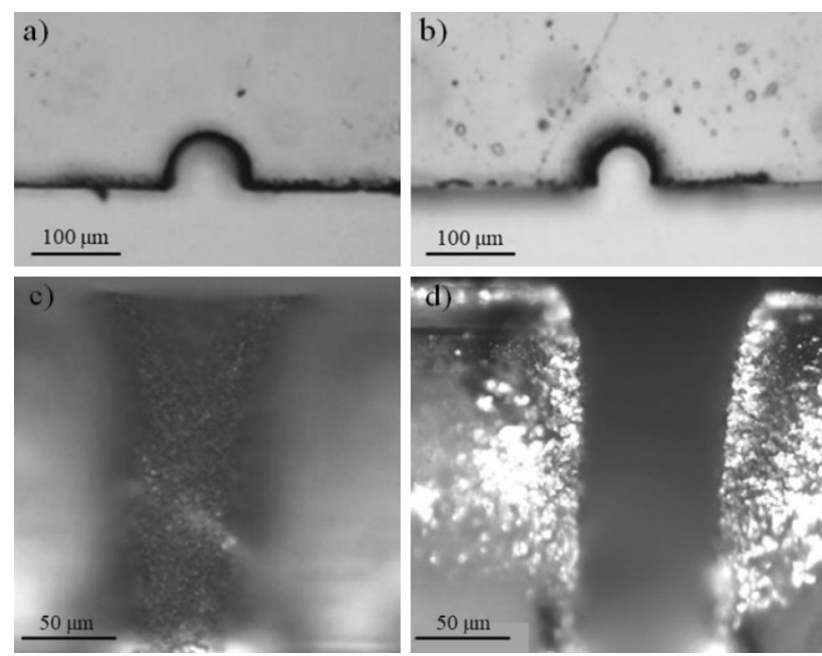

Fig. 9 Optical microscope images of a hole drilled across a lateral edge of the glass sample with fabrication speed $2.8 \mathrm{~mm} \mathrm{~s}^{-1}$ and pulse density 5000 pulses $\mathrm{mm}^{-1}$. Imaging of the top surface (a), bottom surface (b), and imaging of the internal side (c) and of the edges (d) of the hole

Table 2 Parameters of the types of BM of the Picoblade laser considered

\begin{tabular}{lllr}
\hline Type of burst & B1 & B2 & B3 \\
\hline Number of pulses & 4 & 4 & 8 \\
$\Delta \mathrm{T}_{B}[\mathrm{~ns}]$ & 12 & 156 & 12 \\
Repetition rate, LRR $[\mathrm{kHz}]$ & 66 & 60 & 60 \\
Burst energy at sample $[\mu \mathrm{J}]$ & 290 & 315 & 320 \\
Pulse energy at sample $[\mu \mathrm{J}]$ & 72.5 & 78.7 & 40 \\
\hline
\end{tabular}

optical microscope. The zoomed images of the internal surface (Fig. 9c) and of the edges (Fig. 9d) provide new information: the internal surface of the hole is smooth, regular and does not present cracks, although the edges present a taper angle of about $20^{\circ}$.

\section{Micromachining in burst mode}

Using the laser source in burst mode allows to irradiate the material with packets of closely spaced pulses instead of single pulses. The parameters of the burst modes initially used in our experiments are shown in Table 2; we can note that the total energy of a burst contains about three times the pulse energy value that we had in the single-pulse mode described before (i.e., about $100 \mu \mathrm{J}$ ), but the energy of a single pulse inside the burst is considerably lower.

\subsection{Comparison between different burst modes}

We started to study the effects of the burst mode by drilling holes in a $200 \mu \mathrm{m}$ AF32 glass sample, with the fabrication parameters initially used in Sect. 3.1 (i.e., fabrication speed $0.8 \mathrm{~mm} \mathrm{~s}^{-1}$, step between writing circles $0.25 \mu \mathrm{m}, 60$ circles, pulse density 5000 pulses $\mathrm{mm}^{-1}$ ), as we did for the single-pulse mode regime. Two types of bursts that mainly differ in the temporal distance $\Delta \tau_{B}$ between the single pulses within the packet were initially tested; in one case, we used the BM type of the Picoblade laser, here denoted as B1, featured by $\Delta \tau_{B}=12 \mathrm{~ns}$, and in another case, we used the BM type denoted by B2 with $\Delta \tau_{B}=156 \mathrm{~ns}$. By working in the burst mode regime, we have been able to drill straightforwardly regular through-holes with a single processing pass, instead of the two passes needed before in the single-pulse writing mode regime. The B1 burst mode led to good quality micro-holes results with no material residues inside the apertures, contrarily to what happened with the B2 case. Note that the fluences are approximately equal for the two burst types regimes, so the outcome of the fabrication depends on the temporal delay between the pulses inside a burst, which may play an important role in the laser-matter interaction. Indeed shockwaves emission occurs in a range of $500 \mathrm{ps}$ to few tens of $\mathrm{ns}$, followed by strong thermal diffusion from the heat-affected zone up to the $\mu$ s time scale [4]. We believe that having more closely spaced pulses within a burst (for a fixed number of pulses), on time scales smaller than $100 \mathrm{~ns}$, allows each of them to interact with hotter and softer matter leading to an increase in the local temperature and emission of a stronger shockwave, therefore probably facilitating the material extrusion during the micromachining process. In any case, material residues and dark halos around the holes disappear after an ultrasonic bath cleaning of the sample in acetone. Figure 10 presents an example of holes drilled in a single processing pass with our BB trepanning technique and the parameters discussed above in the B1 type (top row) and in the $\mathrm{B} 2$ type (bottom row) burst mode regimes, respectively.

\subsection{Effect of the fabrication speed in BM regime}

Once verified that using the burst mode regime allows us to generate in one single machining pass regular throughholes across the AF32 glass sample, we investigated the possibility to increase the fabrication speed. The study was performed with two different burst types and different speed values. In particular, we concentrated on the B1 and B3 burst mode cases (see Table 2) which differ by the number of pulses in the burst, being, respectively, four and eight. The total energy of the two pulse packets are not so different, and the single pulses in the B1 case have 
Fig. 10 Optical microscope images of a hole drilled with 60 writing circles, in one single machining pass, in BM regime with a burst type B1 featured by $\Delta \tau_{B}=12 \mathrm{~ns}$ (top) and with a burst type B2 featured by $\Delta \tau_{B}=156 \mathrm{~ns}$ (bottom). Speed of fabrication $0.8 \mathrm{~mm} . \mathrm{s}^{-1}$. Pulse density 5000 pulses $\mathrm{mm}^{-1}$. $\mathbf{a}$ and $\mathbf{d}$ Top surface, $\mathbf{b}$ and $\mathbf{e}$ bottom surface and $\mathbf{c}$ and $\mathbf{f}$ top surface imaged after $10 \mathrm{~min}$ of ultrasonic bath cleaning of the sample in acetone
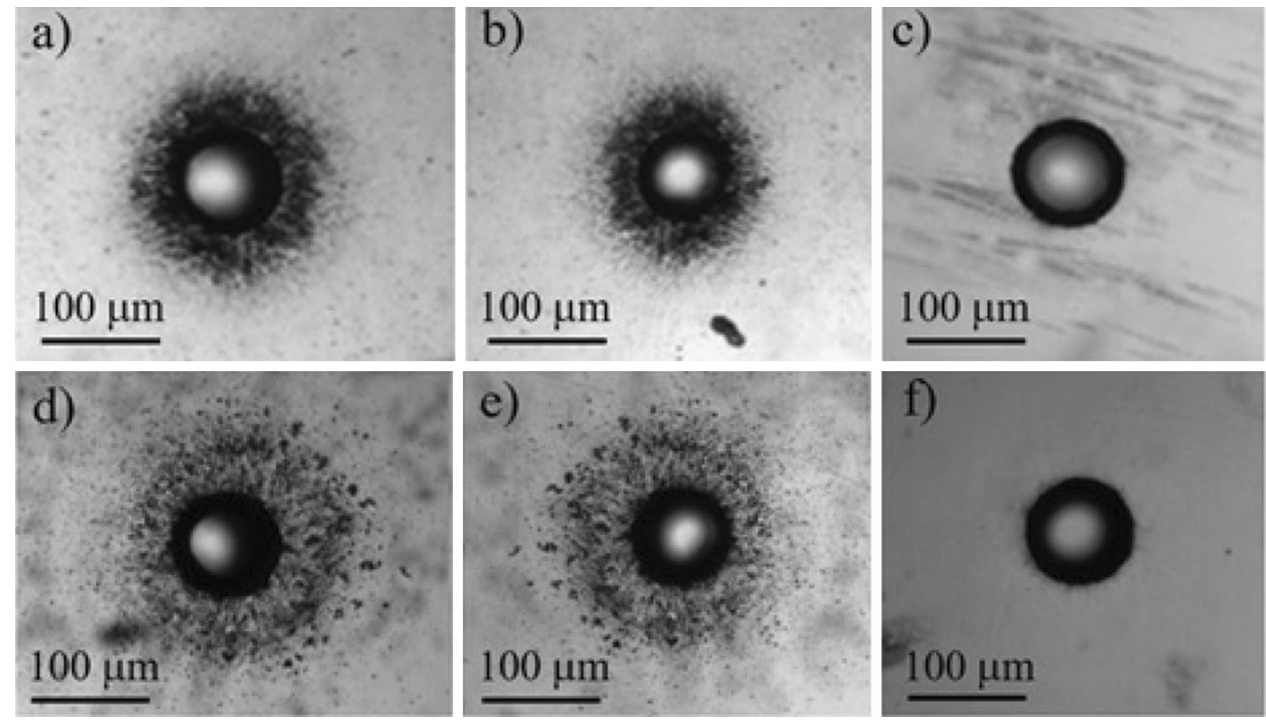
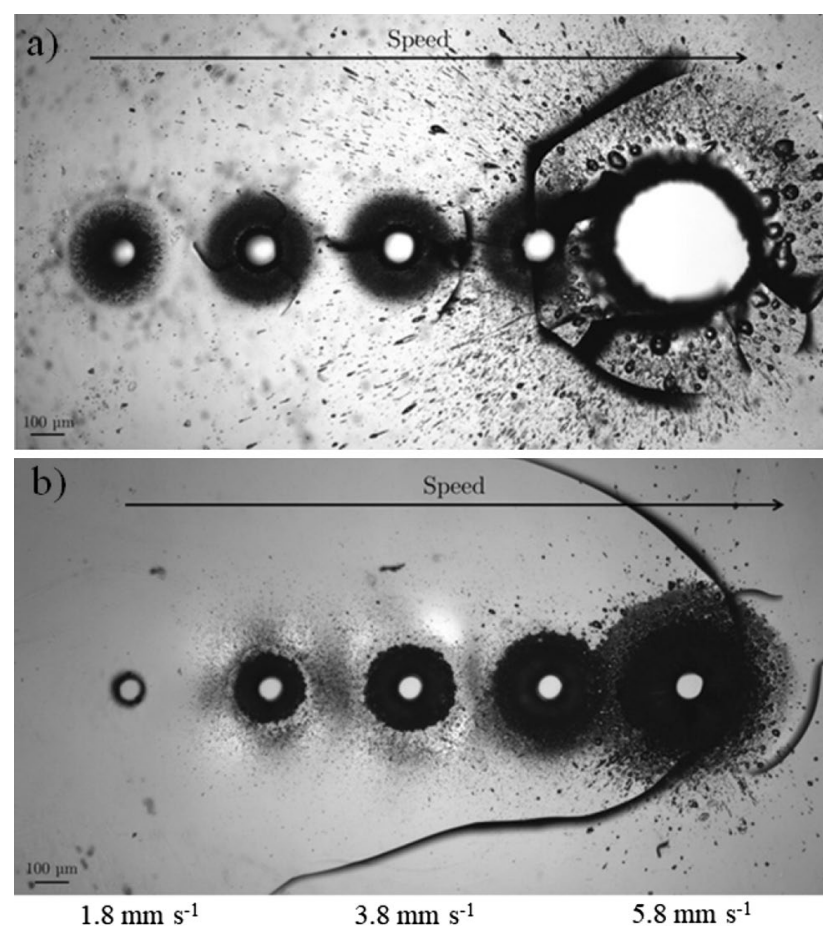

Fig. 11 Optical microscope images of micro-holes drilled with 60 writing circles in one machining pass, with increasing fabrication speeds from $1.8 \mathrm{~mm} \mathrm{~s}^{-1}$ to $5.8 \mathrm{~mm} \mathrm{~s}^{-1}$ by steps of $1 \mathrm{~mm} \mathrm{~s}^{-1}$. Pulse density 5000 pulses $\mathrm{mm}^{-1}$. In a, we used the BM type B1 featured by 4 pulses inside each burst; in $\mathbf{b}$, we used the BM type B3 featured by 8 pulses inside each burst. In both cases, $\Delta \tau_{B}=12 \mathrm{~ns}$. In $\mathbf{b}$, the presented image has been recorded after an ultrasonic bath in acetone

almost twice the energy of the pulses in B3. Figure 11a presents an optical microscope image of the sample top surface after the drilling tests performed in the B1 burst mode regime, for increasing fabrication speeds. Only for a speed value of $1.8 \mathrm{~mm} \mathrm{~s}^{-1}$, the generated hole appears to be regular and with no cracks. For higher values, cracks are present at the surface and they increase in number and in extent, until an explosion occurs in the last case. There, where the fabrication speed is $5.8 \mathrm{~mm} \mathrm{~s}^{-1}$, bubbles surround the aperture, probably due to the high pressure and the vaporized glass [23]. The explosion may also be caused by some defects in the glass. The results obtained with the B3 type burst are shown in Fig. 11 b: the only evident cracks are present in the test with the higher fabrication speed $\left(5.8 \mathrm{~mm} \mathrm{~s}^{-1}\right)$, and small bubbles can be observed. The black halos around the apertures are bigger compared to those of the B1 tests and they do not disappear completely after the ultrasonic bath: probably the melted glass, ejected from the heat-affected zone, has solidified again around the hole. We also note that the through-holes performed with the B1 type burst are more irregular (even if in this BM type, the fluence is lower), and we deduce that the number of pulses contained in the radiation burst can affect the fabrication. More pulses in the packet correspond to a lower energy per single pulse and thus a lower pulse intensity; this implies the generation of fewer free carriers leading to less pronounced thermal effects. For these reasons, even with the Bessel beam machining technique, drilling with a burst containing a greater number of pulses seems to be an advantage for the generation of microholes with a surrounding morphology less affected by thermal effects, in accordance with previous results [24].

\subsection{Influence of pulse energy and fabrication speed}

The results just presented suggest that the energy per packet released to the glass sample in the drilling process with the B1 type burst is too high for obtaining reliable through-holes without cracks on the sample surfaces, 
especially when increasing the fabrication speed. For more completeness, we have studied the influence of the speed of fabrication combined with decreasing values of the bursts energy, in the B1 burst mode type, featured by a number of pulse per burst equal to 4 . In Table 3, we report the fabrication parameters used (speed and burst energy) with the relative fluences calculated as in Sect. 3.3 for an arbitrary time interval of $250 \mu \mathrm{s}$, from Eq. (3), where $N$ is now the number of bursts and $E$ is the total burst energy. Figure 12 shows the results of the micro-drilling test. The fluences reported in blue-green in the table are those for which, for a given speed, the machining has led to through-holes as observed in the images of Fig. 12. Generally, the BB laser machining performed with higher fluences generates through-holes, many of which with cracks. Some cracks cannot be seen because of the dark halos that remain on the surface even after the ultrasonic bath, a drawback in this case of the burst machining regime. We mention that some of the observable cracks

Table 3 Arriving fluences $\left[\times 10^{3} \mathrm{~J} \mathrm{~cm}^{-2}\right]$ during the BB micro-drilling with the B1 type burst, calculated over an arbitrary time interval of $250 \mu \mathrm{s}$, and reported as function of the fabrication speed (bold vertical column) and the packet energy (bold horizontal line)

\begin{tabular}{|c|c|c|c|c|c|}
\hline & \multicolumn{5}{|c|}{ Packet energy $[\mu \mathrm{J}]$} \\
\hline Speed $\left[\mathrm{mm} \mathrm{s}^{-1}\right]$ & 290 & 232 & 174 & 116 & 58 \\
\hline 1.8 & 8.3 & 6.7 & 5.0 & 3.3 & 1.7 \\
\hline 2.8 & 11.8 & 9.5 & 7.1 & 4.7 & 2.3 \\
\hline 5.8 & 19.4 & 15.5 & 11.7 & 7.8 & 3.9 \\
\hline
\end{tabular}

The yellow shaded values correspond to the fluence threshold values for through-hole drilling

Pulse density 5000 pulses $\mathrm{m}^{-1}$. The blue-green data correspond to those leading to through-holes across the $200 \mathrm{~mm}$-thick AF32 glass sample in the images reported in Fig. 12 have appeared or have expanded after the ultrasonic bath: this tells us that after the laser machining the area surrounding the hole is subject to huge stress probably due to the thermal effects, in accordance with what shown in [25].

In order to further comment our results, let us consider the highlighted data in Table 3. In particular, we refer to: "low speed" hole (LSH) the hole performed with speed of $1.8 \mathrm{~mm} \mathrm{~s}^{-1}$ and burst energy of $232 \mu \mathrm{J}$, "medium speed" hole (MSH) the hole performed with speed of $2.8 \mathrm{~mm} \mathrm{~s}^{-1}$ and burst energy of $174 \mu \mathrm{J}$, and "high speed" hole (HSH) the hole performed with speed of $5.8 \mathrm{~mm} \mathrm{~s}^{-1}$ and burst energy of $116 \mu \mathrm{J}$. The relative fluences of these cases (threshold values for through-hole drilling) have been highlighted in yellow in the table. We notice that the value of the threshold fluence necessary to drill a through-hole (green data) seems lower for lower fabrication speeds. In fact, the LSH, which is a through-hole, was performed with a lower fluence than that, respectively, used for the MSH and HSH holes which in fact do not cross the whole sample thickness. We may explain this behavior by considering the intensity of the pulses in the bursts and the temporal distance between two consecutive bursts. The LSH has been machined with the highest burst packet energy, and thus also the highest single-pulse intensity, contrarily to the MSH and the HSH cases where the pulse intensity was lower. A too low intensity could be responsible for a not complete drilling, confirming again the importance of the intensity for an efficient carriers excitation process during the radiation-matter interaction. On the other hand, the MSH and HSH are surrounded by cracks, in contrast to the LSH: having smaller temporal delays between bursts implies larger thermal accumulation effects leading to cracks, as also previously observed in the single-pulse mode, and a less efficient energy deposition along the whole Bessel zone and thus across the
Fig. 12 Optical microscope image (top surface) recorded after an ultrasonic bath in acetone, of micro-holes drilled with 60 writing circles in one machining pass with the $B 1$ burst mode, for three different fabrication speeds (different rows) and for different packet energy values (different columns). Pulse density 5000 pulses $\mathrm{mm}^{-1}$

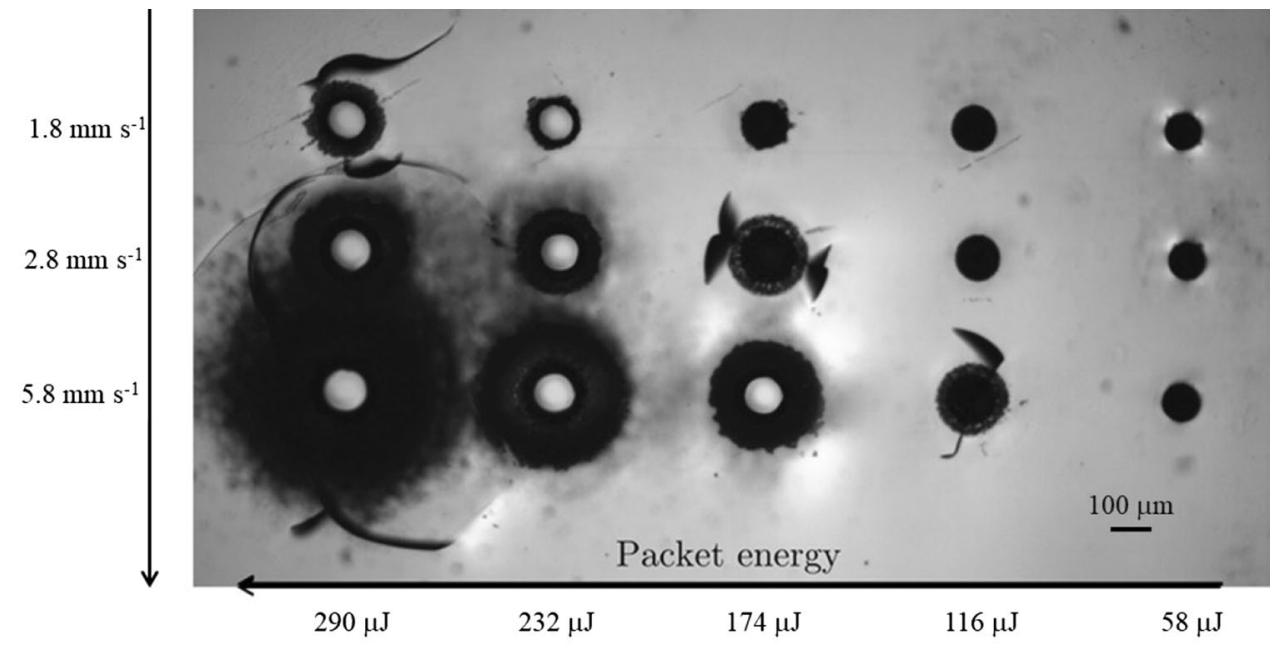

SN Applied Sciences a SPRINGER NatURE journa 

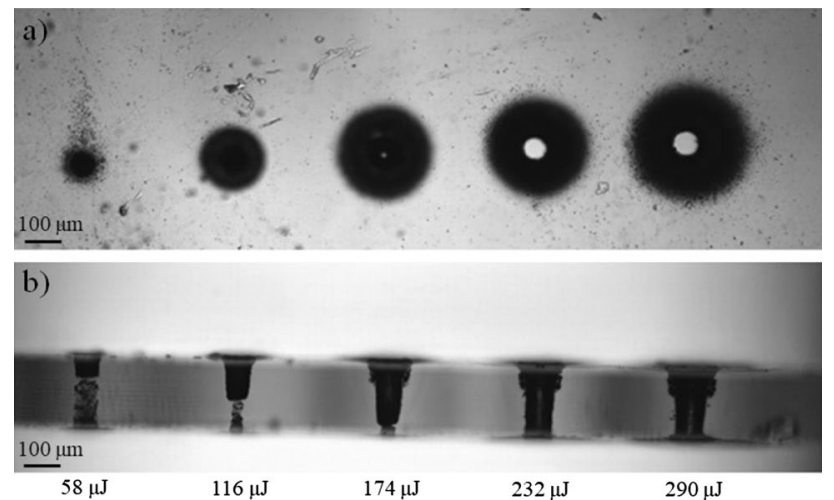

Fig. 13 Optical microscope image (in a top surface and in $\mathbf{b}$ lateral view) of micro-holes drilled with 60 writing circles in one machining pass with the B1 burst mode for increasing packet energy values and a fabrication speed of $2.8 \mathrm{~mm} \mathrm{~s}^{-1}$. Pulse density 5000 pulses $\mathrm{mm}^{-1}$

whole sample thickness. For completeness, we present in Fig. 13 results of micro-holes drilled with a fixed fabrication speed $\left(2.8 \mathrm{~mm} \mathrm{~s}^{-1}\right)$ and increasing packet energies. The optical microscope images show the effect of the BB intensity on the effective radiation-matter interaction inside the glass bulk, especially along the thickness of the sample. At higher intensities, the machining process leads to through-holes even if featured by a wider aperture and a taper in the upper part of the fabrication. The black halos surrounding the holes remain a drawback when using the BM for BB micro-hole drilling at relatively high fabrication speed.

\section{Effect of pulse density: direct comparison between single-pulse mode and BM}

Finally, we have made a direct comparison between the Bessel beam micro-drilling process, respectively, in the single-pulse mode and in the burst mode regime when changing the pulse density of the laser, and using a high fabrication speed. A change in the pulse density affects the spatial and the temporal distributions of the pulses arriving on the sample. In this experiment, we have varied the laser pulse density from 3000 to 5000 pulses $\mathrm{mm}^{-1}$ by steps of 500 pulses $\mathrm{mm}^{-1}$. The holes have been generated in the single-pulse mode with two writing passes (energy per pulse of $100 \mu \mathrm{J}$ ) and in the burst mode (B1) in one pass (energy per packet of $290 \mu \mathrm{J}$ ), with speed of fabrication set to $5.6 \mathrm{~mm} \mathrm{~s}^{-1}$. The results of the laser micromachining are shown in Fig. 14 and the corresponding fluences calculated in the different cases are reported in Table 4. As before, we have used Eq. (3) where $N$ has been taken as the number of bursts occurring in each case.

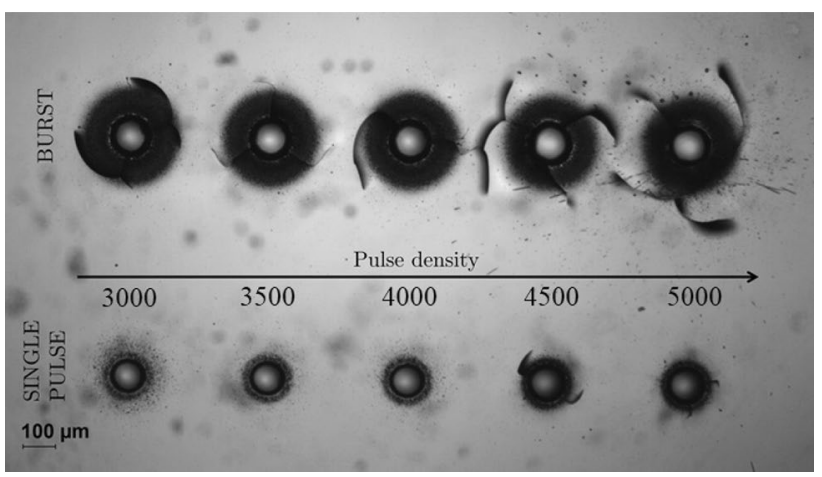

Fig. 14 Optical microscope images of the top surface of a AF32 glass sample. Comparison between the burst mode (B1, one writing pass) and single-pulse mode machining (two writing passes) for micro-hole drilling. The pulse density has been varied from 3000 to 5000 pulses $\mathrm{mm}^{-1}$ by steps of 500 pulses $\mathrm{mm}^{-1}$. The energy values were set to $100 \mu \mathrm{J}$ per pulse in the single-pulse mode and $290 \mu \mathrm{J}$ per packet in the burst mode. Fabrication speed set to $5.6 \mathrm{~mm} \mathrm{~s}^{-1}$

The holes realized with the burst mode present lateral cracks at the surface for every value of the pulse density at the chosen fabrication speed. On the contrary, we observe that the single-pulse mode machining regime allows the drilling of good quality holes for pulse density values smaller or equal than 4000 pulses $\mathrm{mm}^{-1}$ (and fluences lower than $6.1 \times 10^{3} \mathrm{~J} \mathrm{~cm}^{-2}$ ). On one hand in this condition, the fluences available in the burst packets could have been too high, and on the other hand, these results seem to suggest indeed that few tens of $\mu$ s are necessary for a complete cooling down of the heat-affected zone. This last observation is in analogy with that relative to the study shown in Fig. 8, performed in that case for a fixed pulse density at different fabrication speeds. On the other hand, these last tests suggest that faster microdrilling with homogenous results and clean apertures can be achieved by choosing preferentially the single-pulse mode machining regime instead of the $\mathrm{BM}$, and by suitably adjusting the pulse density below a critical level such

Table 4 Arriving fluences during the BB micro-drilling with the B1 type burst, calculated over an arbitrary time interval of $250 \mu \mathrm{s}$, and reported as function of the pulse density used (relatively to the experimental cases of Fig. 15)

\begin{tabular}{llll}
\hline $\begin{array}{l}\text { Pulse density } \\
{\left[\mathrm{mm}^{-1}\right]}\end{array}$ & $\begin{array}{l}\Delta \tau \\
{[\mu \mathrm{s}]}\end{array}$ & \multicolumn{2}{l}{ Fluence $\left[10^{3} \mathrm{~J} \mathrm{~cm}^{-2}\right]$} \\
\cline { 3 - 4 } & & Single pulse & Burst \\
\hline 3000 & 59 & 5.7 & 13.2 \\
3500 & 51 & 5.9 & 13.7 \\
4000 & 45 & 6.1 & 14.3 \\
4500 & 40 & 6.4 & 14.8 \\
5000 & 36 & 6.6 & 15.2 \\
\hline
\end{tabular}

The time interval between the arrival of each pulse is also reported 
to avoid mechanical stresses at the sample surfaces. Note that the lengthy double pass writing process, used here in the single-pulse machining regime, can be avoided by using a higher power laser source, and thus having more pulse energy to distribute along the Bessel zone.

\section{Conclusions}

In this work, we have presented a study of the Bessel beam micro-drilling process on a thin glass sample, namely a 200- $\mu$ m-thick AF32 glass, in different laser micromachining conditions. In particular, we have used a high-repetitionrate pulsed laser source allowing to work in the singlepulse and in the burst mode processing regime, respectively. This has allowed us to extend previous preliminary results on hole drilling by means of this technique, and in particular to analyze the differences, with advantages or drawbacks, of these two machining regimes.

The microfabrication has been performed in the socalled density mode regime, that is by keeping fixed the number of pulses $\mathrm{mm}^{-1}$ arriving on the sample. Different machining parameters have been varied with the consequent observation by optical microscopy, of the morphology of the holes drilled. An analysis of the quality of the through micro-holes generated in double pass, with the standard single-pulse fabrication regime, has been performed as a function of the number of writing circles of the trepanning process, showing the dependence of the hole diameters on this number. We have also mentioned the existence of a minimum number of circular writing trajectories related to the thickness of the sample, for the generation of a homogeneous cylindrical throughhole. The dependence on the fabrication speed has also been studied by keeping the pulse density fixed to 5000 pulses $\mathrm{mm}^{-1}$. The tests have evidenced the occurrence of cracks and stress at the sample surfaces when higher speeds (implying a reduction of the time interval between a pulse and the other) are considered, due to possible thermal accumulation effects. In our case in these conditions, a maximum fabrication speed of $2.8 \mathrm{~mm} \mathrm{~s}^{-1}$ could be used to produce regular through-holes without cracks around the apertures.

The micro-drilling experiments performed in burst mode have highlighted a stronger influence of the fabrication speed on the quality of the through-holes generated, for the same mode density as before ( 5000 pulses $\mathrm{mm}^{-1}$ ). In particular, with the BM types featured by a smaller number of pulses inside the packet, evident thermal effects manifesting with strong cracks could be observed. On the other hand with respect to the single mode machining regime, it is possible for a fixed pulse density to drill through-holes in the BM regime with higher fabrication speed and thus with a faster machining process, provided a lower burst energy is considered. Our results seem to indicate that thermal effects leading to dark halos around the holes remain nevertheless the drawback of the BM regime machining, and suitable energy and pulse density parameters must be chosen for a careful optimization of the quality of the micro-hole to be drilled. In particular, we have shown that by reducing the pulse density, more regular through-holes and cleaner apertures at the sample surfaces can be in fact more effectively generated (with a larger fabrication speed) in the single-pulse fabrication regime than in the burst mode regime. In other words, it is important to adjust the pulse density below a critical level such to avoid mechanical stresses at the sample surfaces.

All this suggests that the choice of the machining regime to be used for BB micro-hole drilling (i.e., the single-pulse or the BM machining regime) depends on the target (better quality, no cracks, higher fabrication speed) one would like to reach. To conclude, BM is advantageous with respect to the single-pulse mode fabrication in terms of lower energy per pulse needed and higher speed of drilling. The drawback of the BM is the presence of stronger thermal effects leading to potential surface cracks. Finally, our results confirm that the interaction of the laser beam with the material is not only influenced by the incident beam fluence, but also by the intensity of the single pulses and the temporal and spatial distances between these or between the bursts, and that these parameters play an important role in the outcome of the machining.

Acknowledgements We thank Altechna Rnd. for the access to the Picoblade2 laser source. OJ thanks Davide Valetti for useful discussions.

\section{Compliance with ethical standards}

Conflict of interest On behalf of all authors, the corresponding author states that there is no conflict of interest.

\section{References}

1. Sibbett W, Lagatsky AA, Brown CTA (2012) The development and application of femtosecond laser systems. Opt Express 20:6989

2. Malinauskas M, Žukauskas A, Hasegawa S, Hayasaki Y, Mizeikis V, Buividas R, Juodkazis S (2016) Ultrafast laser processing of materials: from science to industry. Light Sci Appl 5:e16133

3. Gross S, Withford MJ (2015) Ultrafast-laser-inscribed 3D integrated photonics: challenges and emerging applications. Nanophotonics 4:332-352

4. Gattass R, Mazur E (2008) Femtosecond laser micromachining in transparent materials. Nat Photonics 2:219

5. Mottay E, Liu X, Zhang H, Mazur E, Sanatinia R, Pfleging W (2016) Industrial applications of ultrafast laser processing. MRS Bull 41(12):984-992 
6. Gattass RR, Cerami LR, Mazur E (2006) Micromachining of bulk glass with bursts of femtosecond laser pulses at variable repetition rates. Opt Express 14(12):5279-5284

7. Knappe R, Haloui H, Seifert A, Weis A, Nebel A (2010) Scaling ablation rates for picosecond lasers using burst micromachining. In: Proceedings of SPIE 7585:75850H-7585-6

8. Esser D, Rezaei S, Li J, Herman PR, Gottmann J (2011) Time dynamics of burst-train filamentation assisted femtosecond laser machining in glasses. Opt Express 25:25632

9. Durnin J, Miceli JJ Jr, Eberly JH (1987) Diffraction-free beams. Phys Rev Lett 58:1499

10. McGloin D, Dholakia K (2005) Bessel beams: diffraction in a new light. Contemp Phys 46:15-28

11. Bhuyan MK, Jedrkiewicz O, Sabonis V, Mikutis M, Recchia S, Aprea A, Bollani M, Di Trapani P (2015) High-speed laser-assisted cutting of strong transparent materials using picosecond Bessel beams. Appl Phys A 120:443-446

12. Rapp L, Meyer R, Furfaro L, Billet C, Giust R, Courvoisier F (2017) High speed cleaving of crystals with ultrafast Bessel beams. Opt Express 25:9312

13. Dudutis J, Stonys R, Račiukaitis J, Gečys P (2018) Bessel beam asymmetry control for glass dicing applications. Procedia CIRP 74:333-338

14. Meyer R, Froehly L, Giust R, Del Hoyo J, Furfaro L, Billet C, Courvoisier $F$ (2019) Extremely high-aspect-ratio ultrafast Bessel beam generation and stealth dicing of multi-millimeter thick glass. Appl Phys Lett 114:201105

15. Jedrkiewicz O, Valetti D, Di Trapani P (2019) Etching and drilling of through-holes in thin glass by means of picosecond Bessel beams. SN Appl Sci 1:1267

16. Bhuyan MK, Courvoisier F, Lacourt PA, Jacquot $M$, Salut $R$, Furfaro L, Dudley JM (2010) High aspect ratio nanochannel machining using single shot femtosecond Bessel beams. Appl Phys Lett 97:081102

17. Bhuyan MK, Courvoisier F, Lacourt PA, Jacquot M, Furfaro L, Withford MJ, Dudley JM (2010) High aspect ratio taper-free microchannel fabrication using femtosecond Bessel beams. Opt Express 18:566

18. Bhuyan MK, Courvoisier F, Phing HS, Jedrkiewicz O, Recchia S, Di Trapani P, Dudley JM (2011) Laser micro- and nanostructuring using femtosecond Bessel beams. Eur Phys J Spec Top 199:101

19. Duocastella M, Arnold CB (2012) Bessel and annular beams for material processing. Laser Photonics Rev 6:607

20. Garzillo V, Jukna V, Couairon A, Grigutis R, Di Trapani P, Jedrkiewicz O (2016) Optimization of laser energy deposition for singleshot high aspect-ratio microstructuring of thick BK7 glass. J Appl Phys 120:013102

21. Liu X, Sanner N, Sentis M, Stoian R, Zhao W, Cheng G, Utéza O (2018) Front-surface fabrication of moderate aspect ratio microchannels in fused silica by single picosecond Gaussian-Bessel laser pulse. Appl Phys A 124:206

22. Polesana P, Franco M, Couairon A, Faccio D, Di Trapani P (2018) Filamentation in Kerr media from pulsed Bessel beams. Phys Rev A 77:043814

23. Tan D et al (2016) Femtosecond laser induced phenomena in transparent solid materials: fundamentals and applications. Prog Mater Sci 76:154-228

24. Javaux C et al. (2015) Effects of burst mode on transparent material processing. In: Proceedings of SPIE 9351, laser-based microand nanoprocessing IX, 93510M

25. Mishchik K et al (2017) Improved laser glass cutting by spatiotemporal control of energy deposition using bursts of femtosecond pulses. Opt Express 25:33271

Publisher's Note Springer Nature remains neutral with regard to jurisdictional claims in published maps and institutional affiliations. 